\title{
HEAT TRANSFER IN ROUGH MICROCHANNELS UNDER RAREFIED FLOW CONDITIONS
}

\author{
Croce G. ${ }^{1}$ and Rovenskaya O.I. ${ }^{2}$ \\ *Author for correspondence \\ ${ }^{1}$ DIEG, \\ University of Udine \\ Udine, 33100, Italy \\ E-mail: giulio.croce@uniud.it \\ ${ }^{2}$ Dorodnicyn Computing Centre of Russian Academy of Sciences \\ Vavilova st. 40, 119333 \\ Moscow, Russia
}

\begin{abstract}
A hybrid solver dynamically coupling kinetic solutions computed in local rarefied areas and Navier-Stokes solutions in the rest of the flow is used for the analysis of heat transfer in a rough microchannel. Roughness geometry is modeled as a series of triangular obstructions and a relative roughness up to $5 \%$ of the channel height is considered. Wide range of the Knudsen numbers (from 0.01 up to 0.1 ) is considered, at low Mach number (nearly incompressible flow). The competition between roughness, rarefaction and heat transfer effects is discussed in terms of averaged Nusselt and Poiseuille numbers and mass flow rate. Discrepancy between the full NavierStokes and hybrid solutions is investigated, assessing the range of applicability of the first order slip boundary condition for rough geometries with and without heat transfer.
\end{abstract}

\section{INTRODUCTION}

A considerable amount of research activity in the last decade has been devoted to the understanding of small scale fluid and heat transfer phenomena. In particular, gas flows, such as those in micro heat exchangers and micro fluid machines, pose several specific problems, from the onset of rarefaction related phenomena to the presence of multi-scale relevant features.

Navier-Stokes equations, in fact, work perfectly for macroscale flows, but at micro-scale may become inaccurate in local rarefied areas. Such inconsistency can be partially overcome by applying appropriate slip boundary conditions. However, as indicated in literature, the first order slip condition is valid only for local Knudsen number ( $K n$, ratio between mean free path and relevant geometrical scale) less than 0.1 , and any attempt to increase its range, resorting to higher order slip boundary conditions, is not trivial and highly geometrical dependent. On the other hand, micro-scales are accurately described by more general, but much more time and memory consuming kinetic equations, which require a discretization in both physical and velocity spaces. However, within the range of Knudsen number typical for MEMS devices, the flow can usually be subdivided into a local rarefied regime area along the walls and an internal core, where the flow behaves as a continuum. Therefore, a multi-scale hybrid continuum/kinetic solver, capable of an accurate, yet efficient simulation of multi-scales flows, offers a promising engineering approach and an up to date topic in scientific community [1-3].

Such a hybrid scheme, using a dynamically updated decomposition of the physical space into kinetic and continuum sub-domains identified via a gradient-based Knudsen number, was described and successfully applied to the simulation of gas flow through a slit in [4]. The solver reliability for near wall modelling was later demonstrated in [5] via simulation of the gas flow through a channel of finite length for a wide range of $K n$ number and pressure ratios.

The flow over a rough surface is an example of engineering problem where the combination of kinetic and continuum scale features plays a significant role. In microchannel analysis, in fact, the Knudsen number is defined using the hydraulic diameter as relevant geometrical scale. However, it is well known that in MEMS fabrication, due to the small scale, it is unlikely to create a perfectly smooth surface. Thus, if roughness effect is the object of the analysis, the relevant geometrical scale is related to the roughness details rather than to the hydraulic diameter, and rarefaction is important even if the diameter based $K n$ is relatively high. Pure Navier-Stokes equations in principle should thus be limited to flows where the roughness size, rather than the diameter, is much smaller than the mean free path of the gas. As was shown in [6] this condition may not be fulfilled in application of practical interest; thus, Navier-Stokes approach does not allow to obtain correct results for microchannel pressure losses even if most of the channel core flow is safely in the continuum regime.

On the other hand the application of the hybrid solver, implementing a kinetic approach near the roughness surface, provides results in a better agreement with fully kinetic ones at a much lower computational cost [6].

Compressibility and rarefaction effects on the heat transfer in rough microchannel has been analysed in [7] via NavierStokes solver. Highly rarefied flows (Knudsen numbers from 0.02 to 0.12 ) were computed in [9] via direct Monte Carlo simulations in very rough geometries, from $5 \%$ up to $12 \%$, while the low Mach number level prevented any compressibility effect. A pure kinetic solver based on the solution of the S-model equation was used in [10] for wide 
ranges of Mach (up to choked flow) and Knudsen numbers (from slip to transition regime), demonstrating a significant influence of surface roughness, rarefaction and compressibility on the friction factor.

Nowadays, little is still found in the literature for the investigation of roughness effect in the presence of heat transfer for a relatively higher value of $K n$, lying close to the border of continuum hypothesis validity (i.e., in the slip-flow regime). For example, in $[7,8,11]$ using Navier-Stokes equations a noticeable effect of roughness on Nusselt number has been demonstrated, with a reduction in $\mathrm{Nu}$ with increasing Knudsen number, while the value of $K n$ number was kept relatively low. Even among the most recent papers [12,13] the continuum-slip approach is usually followed, and focus is shifted on the possible roughness shapes.

Here, the hybrid solver [6] is applied to the analysis of the effect of the surface roughness on the heat transfer in gaseous flow: thus, the actual reliability of the first order slip boundary condition for the simulation of flow along rough fine-textured surfaces, with and without a heat transfer, is estimated.

\section{NOMENCLATURE}

\begin{tabular}{|c|c|c|}
\hline $\mathbf{c}=\xi-\mathbf{V}$ & {$[\mathrm{m} / \mathrm{s}]$} & $\begin{array}{l}\text { Relative speed of a single particle against a } \\
\text { background gas }\end{array}$ \\
\hline$D_{H}$ & {$[\mathrm{~m}]$} & Hydraulic diameter \\
\hline$e_{t o t}$ & {$\left[\mathrm{~J} / \mathrm{m}^{3}\right]$} & Macroscopic total energy \\
\hline$e_{i n t}$ & {$[\mathrm{~J} / \mathrm{kg}]$} & Macroscopic internal energy \\
\hline$f(t, \mathbf{x}, \xi)$ & {$\left[\mathrm{s}^{3} / \mathrm{m}^{6}\right]$} & Particle velocity distribution function \\
\hline$f$ & {$[-]$} & Friction coefficient, dimensionless \\
\hline$F(\mathbf{U})$ & {$[-]$} & Flux \\
\hline$h$ & {$\left[\mathrm{~W} /\left(\mathrm{m}^{2} \mathrm{~K}\right)\right]$} & Local heat transfer coefficient \\
\hline$H$ & {$[\mathrm{~m}]$} & Height of the channel \\
\hline$k$ & $\begin{array}{l}{\left[1.38 .10^{-}\right.} \\
\left.{ }^{23} \mathrm{~J} / \mathrm{K}\right]\end{array}$ & Boltzmann constant \\
\hline$K n$ & {$[-]$} & Knudsen number, dimensionless \\
\hline$M$ & {$\left[\mathrm{~s}^{3} / \mathrm{m}^{6}\right]$} & Maxwellian distribution function \\
\hline$m$ & {$[\mathrm{~kg}]$} & mass of the molecule \\
\hline$n$ & {$\left[1 / \mathrm{m}^{3}\right]$} & local number density \\
\hline$N u$ & {$[-]$} & Nusselt number \\
\hline$p$ & {$[\mathrm{~Pa}]$} & pressure \\
\hline Po & {$[-]$} & Poiseuille number, dimensionless \\
\hline q & {$\left[\mathrm{W} / \mathrm{m}^{2}\right]$} & Heat flux vector \\
\hline $\mathrm{R}=k / m$ & $\begin{array}{l}{[8.3145} \\
\mathrm{J} / \mathrm{mol} \mathrm{K}]\end{array}$ & ideal gas constant \\
\hline$s$ & {$[\mathrm{~m}]$} & Distance between peaks \\
\hline$t$ & {$[\mathrm{~s}]$} & Time \\
\hline$T$ & {$[\mathrm{~K}]$} & Temperature \\
\hline $\boldsymbol{U}$ & & Vector of conservative macroscopic variables \\
\hline $\mathbf{V}=(u, v, w)$ & {$[\mathrm{m} / \mathrm{s}]$} & Gas bulk velocity vector \\
\hline $\mathbf{x}=(x, y, z)$ & {$[\mathrm{m}]$} & Position vector \\
\hline$W$ & [ ] & Mass flow rate \\
\hline
\end{tabular}

Special characters

$\begin{array}{ll}\gamma & {[-]} \\ \Gamma_{\mathrm{c}} & {[\mathrm{m}]} \\ \delta_{i j} & {[-]} \\ \varepsilon & {[-]} \\ \eta(\mathbf{x}) & {[\mathrm{m}]} \\ \kappa & {[\mathrm{W} / \mathrm{m} \mathrm{K}]} \\ \lambda & {[\mathrm{m}]} \\ \mu & {[\mathrm{kg} / \mathrm{m} \mathrm{s}]} \\ \xi & {[\mathrm{m} / \mathrm{s}]} \\ \xi_{x}, \xi_{y}, \xi_{z} & {[\mathrm{~m} / \mathrm{s}]} \\ \rho & {\left[\mathrm{kg} / \mathrm{m}^{3}\right]} \\ \Omega_{\mathrm{K}} & {\left[\mathrm{m}^{2}\right]} \\ \Omega_{\mathrm{NS}} & {\left[\mathrm{m}^{2}\right]}\end{array}$

Specific heat ratio, dimensionless coupling boundary

Kronecker delta

Relative roughness, dimensionless

Outward normal vector

Thermal conductivity

Mean free path

Gas viscosity

Particle velocity vector

Components of particle velocity vector

Density

Kinetic domain

Navier-Stokes domain

$\begin{array}{ll}\text { Subscripts } & \\ a v & \text { Averaged between inlet and outlet values } \\ b & \text { Bulk value } \\ C E & \text { Chapman-Enskog } \\ c & \text { Coupling } \\ e & \text { Exit } \\ i & \text { Incoming } \\ i n t & \text { Internal } \\ i s & \text { Isentropic } \\ \mathrm{K} & \text { Kinetic } \\ l & \text { Local value } \\ o & \text { Outcoming } \\ \text { tot } & \text { Total } \\ w & \text { Wall } \\ 0 & \text { Total value, equilibrium state }\end{array}$

\section{STATEMENT OF THE PROBLEM}

We consider a planar microchannel of width $H$ and length $l=10 H$, connecting two reservoirs of size $L_{x} \times L_{y}$, as represented in Figure 1. Flow is symmetric about $y=0$, allowing simulation of only a half of the domain. The monatomic gas in the reservoirs far from the channel is in equilibrium at constant pressures $p_{0}$ in the inlet reservoir and $p_{e}$ in the outlet $\left(p_{0}>p_{e}\right)$, and temperature $T_{0}$. The walls are assumed at a fixed temperature $T_{w}$. The wall roughness, shown in Fig. 2, is modelled as a series of triangular obstructions with a sharp angle of $45^{\circ}$. The height of a single peak is $h=\varepsilon H$ and the distance between peaks is $s=5 h$. Several relative roughness heights $\varepsilon$ have been considered: $1.25 \%, 2.5 \%$ and $5 \%$. For sake of computational efficiency only a small channel part of length $L_{r}=3.15 H$ placed near the channel exit is modelled as a rough one [6], while the rest of the channel and reservoirs walls are smooth, as in Fig. 1. Hence, the rough region includes 12 roughness ridges for $\varepsilon=5 \%, 24$ ridges for $\varepsilon=2.5 \%$ and 48 ridges for $\varepsilon=1.25 \%$. At low pressure ratio (low Ma), the inlet smooth region allows for fully developed flow approaching the rough region. It should be noticed that the interface $I_{c}$ between kinetic and NS sub-domains is dynamically updated during the computation.

The inlet/outlet boundary conditions are standard and specify the inlet total temperature $T_{0}$, total pressure $p_{0}$ and flow direction, and the static pressure $p_{e}$ at the outlet $\left(p_{0}>p_{e}\right)$ :

$$
\begin{aligned}
& \frac{p_{0}}{p_{e}}=\left(1+\frac{\gamma-1}{2} M a_{i s}^{2}\right)^{\gamma /(\gamma-1)} \\
& \frac{T_{0}}{T_{e}}=\left(1+\frac{\gamma-1}{2} M a_{i s}^{2}\right) \\
& \rho_{0}=p_{0} / R T_{0}
\end{aligned}
$$

where $M a_{i s}$ is the isentropic exit Mach number, i.e. $M a$ that would arise from an isentropic flow with the same pressure ratio as the real one.

The monatomic gas flows due to a pressure difference between upstream and downstream reservoirs. The static inlet pressure $p_{i}$ is the result of computation, although, due to the low inlet velocities, it almost coincides with inlet total pressure $p_{0}$. Rarefaction of gas flow is determined by Knudsen number $K n$ :

$$
K n=\frac{\lambda}{H}
$$


where the local mean free path $\lambda$ for the hard-sphere molecular model, using in the present study, is defined as:

$$
\lambda=\frac{16 \mu}{5 \rho} \sqrt{\frac{m}{2 \pi k T}}
$$

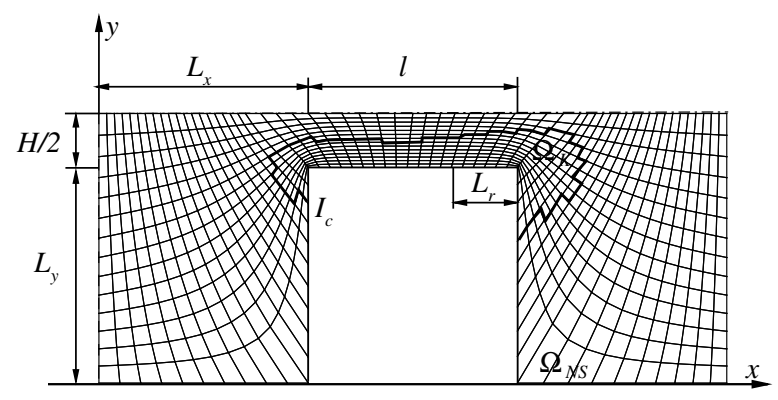

Figure 1 Sketch of computational domain

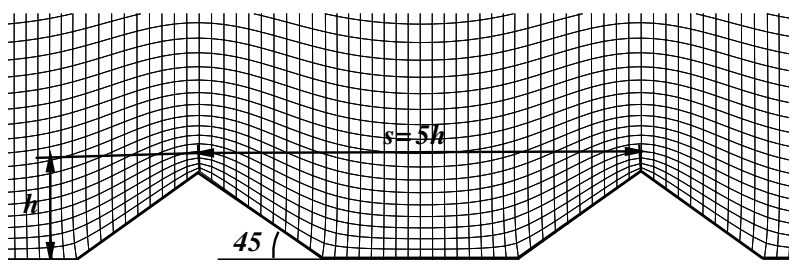

Figure 2 Rough elements details

\section{GLOBAL AND LOCAL FLOW PERFORMANCES}

The channel global and local performance are presented in terms of the Poiseuille number, Nusselt number and mass flow rate. The mass flow rate through the channel is non dimensionalized using the analytical mass flow rate in the limit of free molecular regime $\dot{m}_{f m}$ :

$$
W=\frac{\dot{m}}{\dot{m}_{f m}} \quad \dot{m}_{f m}=\frac{H p_{0}}{\sqrt{\pi} v_{0}},
$$

where $\dot{m}$ is computed as :

$$
\dot{m}=2 \int_{-H / 2}^{0} \rho(x, y) u(x, y) d y .
$$

The friction factor $f_{r}$ is defined as an averaged value either over a portion of a rough channel section or over a single geometrically periodic roughness element of length $s$. In both cases, $f_{r}$ is computed as following:

$$
f_{r}=2 \frac{\Delta \bar{p}}{\Delta L} \frac{D_{H}}{\bar{\rho}_{a v} \bar{u}_{a v}{ }^{2}}=2 \frac{\Delta \bar{p}}{\Delta L} \frac{D_{H} \bar{\rho}_{a v}}{\overline{\dot{m}}_{a v}{ }^{2}},
$$

where the hydraulic diameter $D_{H}=2 H$, overbar means averaged values over cross section, $\Delta$ and $a v$ are the difference and the averaged between inlet and outlet values. The Poiseuille number $P o_{\varepsilon}$ is then written in terms of the local $R e$ as:

$$
\begin{aligned}
& P o_{\varepsilon}=f_{r} \cdot \operatorname{Re}=2 \frac{\Delta \bar{p}}{\Delta L} \frac{D_{H}^{2}}{\bar{\mu}_{a v} \bar{u}_{a v}}=2 \frac{\Delta \bar{p}}{\Delta L} \frac{\bar{\rho}_{a v} D_{H}^{2}}{\bar{\mu}_{a v} \overline{\dot{m}}_{a v}}, \\
& \operatorname{Re}=\frac{\bar{\rho}_{a v} \bar{u}_{a v} D_{h}}{\bar{\mu}_{a v}}=\frac{\overline{\dot{m}}_{a v} D_{H}}{\bar{\mu}_{a v}} .
\end{aligned}
$$

Due to the mass conservation, $R e$ changes only slightly along the channel, because of the $\mu$ variation with temperature. Global $P o$ number is computed between the inlet and outlet section of a rough sector $L_{r}$ of length $L^{*}=2.25 H$, (i.e., skipping small regions both at the inlet and exit of a rough sector, to avoid entrance and exit effects), including 9, 18 and 42 rough modules, for relative roughness height $\varepsilon=5 \%, 2.5 \%$ and $1.25 \%$, respectively. Global values are computed over the same size section $L^{*}$ also for the smooth channel case, $\varepsilon=0$.

A common performance parameter for the heat transfer in micro/macro channels is the Nusselt number: if $h$ is the local heat transfer coefficient, we may define a local Nusselt as

$$
N u_{l}=\frac{h D_{H}}{\kappa}=\frac{q(x) D_{H}}{\kappa\left(T_{w}-T_{b}(x)\right)}
$$

where $q$ is the heat flux, $\kappa$ is the thermal conductivity, $T_{b}$ is the bulk flow temperature. Global Nusselt number $N u_{\varepsilon}$ is computed by averaging over a rough/smooth channel section $L^{*}$ as:

$$
N u_{\varepsilon}=\frac{1}{L^{*}} \int_{L^{*}} N u(x) d x
$$

\section{HYBRID METHOD}

The main challenge in the development of hybrid solver is the identification of kinetic and continuum domains, as well as the choice of a proper coupling between these domains. One of the advantages of the present hybrid algorithm is that it allows to couple existing in-house codes for the numerical solution of the Boltzmann kinetic (and its model) [14] and Navier-Stokes equations [7].

The choice of breakdown criterion and its threshold value, defining the size and position of kinetic domains, is important: an underestimated kinetic domain leads to incorrect numerical results $[4,5]$, an overestimated one reduces the advantage in computational efficiency. The most robust and popular criterion is a local, gradient-length Knudsen number $K n_{G L}(\mathbf{x})$ [3-5]:

$$
\begin{aligned}
& K n_{G L}(\mathbf{x})=\max \left(K n_{G L \rho}, K n_{G L V}, K n_{G L T}\right), \\
& K n_{G L \Phi}(\mathbf{x})=K n \frac{|\nabla \Phi|}{\Phi}
\end{aligned}
$$

where $K n$ is the local Knudsen number (eq. 4$), \Phi=(\rho,|\mathbf{V}|, T)$ the vector of local flow parameters. The kinetic solver is activated when $K n_{G L}$ is larger than the threshold value $\Xi$. As shown in $[4,5]$, a threshold value $\Xi=0.1$ already guaranteed a difference between hybrid and kinetic solutions within $1 \%$.

The condition $\mathrm{Kn}_{G L}(\mathbf{x}) \geq \Xi$ identifies the kinetic domain $\Omega_{\mathrm{K}}$, where the S-model kinetic equation [15] is solved :

$$
\begin{aligned}
& \frac{\partial f}{\partial t}+\xi \frac{\partial f}{\partial \mathbf{x}}=J_{S}(f, f)=\frac{p}{\mu}(S-f) \\
& S(\rho, \mathbf{c}, T)=M(\rho, \mathbf{c}, T)\left(1+\frac{2 m^{2} \mathbf{q c}}{15 \rho(k T)^{2}}\left(\frac{m \mathbf{c}^{2}}{2 k T}-\frac{5}{2}\right)\right) \\
& M(\rho, \mathbf{c}, T)=\frac{\rho}{(2 \pi R T)^{3 / 2}} \exp \left(-\frac{\mathbf{c}^{2}}{2 R T}\right)
\end{aligned}
$$

where $f=f(t, \mathbf{x}, \xi)$ is the velocity distribution function, i.e. the probability of finding a molecule with velocity $\xi=\left(\xi_{x}, \xi_{y}, \xi_{z}\right) \in \mathrm{R}^{3}$ in the position $\mathbf{x}=(x, y, z)$ at the time $t$. 
$S(\rho, \mathbf{c}, T)$ and $M(\rho, \mathbf{c}, T)$ are the standard local Shakhov and Maxwell distribution functions, respectively.

The solution on the continuum domain, where $K n_{G L}(\mathbf{x})<\Xi$, is computed from viscous, compressible two-dimensional NS equations written in terms of conservative variables as:

$$
\begin{aligned}
& \frac{\partial \mathbf{U}}{\partial t}+\frac{\partial \mathbf{F}(\mathbf{U})}{\partial \mathbf{x}}=0, \\
& \mathbf{U}=\left(\rho, \rho \mathbf{V}, \rho e_{t o t}\right), \quad e_{t o t}=e+\mathbf{V}^{2} / 2
\end{aligned}
$$

where $e_{t o t}$ is the total energy per unit mass, $\mathbf{U}$ is the vector of macroscopic values, $\mathbf{F}(\mathbf{U})$ is the flux vector, including the convective (inviscid) and diffusive (viscous) components.

In the kinetic description of the gas flow the macroscopic density, momentum and internal energy per unit mass and the heat fluxes are defined by integration over whole velocity space $\mathrm{R}^{3} \in[-\infty ; \infty]$ as following:

$$
\begin{aligned}
& \rho=\int f d \xi \rho(u, v)^{T}=\int\left(\xi_{x}, \xi_{y}\right)^{T} f d \xi \rho e=\frac{1}{2} \int \mathbf{c}^{2} f d \xi \\
& \left(q_{x}, q_{y}\right)^{T}=m \int\left(c_{x}, c_{y}\right)^{T} \mathbf{c}^{2} f d \xi
\end{aligned}
$$

Since the flow is two-dimensional it is possible to eliminate the third velocity component in a model equation $[4,15]$. The $\mathrm{S}$ - model equation is discretized in both velocity and physical spaces and solved using the explicit-implicit numerical scheme $[4,15]$. The transport term in equation (15) is treated explicitly and approximated by a standard finite volume scheme. In particular, the numerical fluxes are determined by the standard finite volume TVD scheme with minmod limiter [12].

Navier-Stokes solver is based on a hybrid finite differencefinite volume method with second order accuracy in space and time $[4,17]$. The solution is advanced in time via Crank Nicolson integration scheme.

The coupling between NS and kinetic solutions is achieved by matching half fluxes of mass, momentum and energy at the sub-domains interface $I_{c}$, thus taking care of the conservation of mass, momentum and energy through the interface [4].On the interface, the NS model acts on the kinetic one imposing a standard Chapman-Enskog (CE) velocity distribution function for the particles coming into the kinetic sub-domain $\Omega_{\mathrm{K}}[4,18]$, while fluxes of mass, momentum and energy predicted by the kinetic solver are imposed into NS sub-domain $\Omega_{\mathrm{NS}}$.

The interface $I_{c}$ position is recomputed at each time step; if a node considered as continuum (NS) at previous time step becomes a kinetic ones at current time step, the kinetic distribution function is initialized as the CE distribution function.

Boundary conditions in the continuum domain are specified according to eqs. (1-3). In the kinetic domain, at inlet/exit boundaries Maxwell velocity distribution function is assumed for incoming particles with density, temperature and velocity specified in the inlet/outlet reservoirs. At solid wall in $\Omega_{\mathrm{K}}$ domain the Maxwell diffuse reflecting boundary condition with the full accommodation is applied [4], while in $\Omega_{\mathrm{NS}}$ subdomain the first order slip and Smoluchowski temperature jump boundary conditions are used. The hybrid code is parallelized in order to improve its efficiency using MPI message passing protocol.

The problem is recast in terms of non-dimensional variables using inlet reservoir equilibrium values as reference ones: density $\rho_{0}$, temperature $T_{0}$, reference dynamic viscosity $\mu_{0}$, most probable velocity $v_{0}=\left(2 \mathrm{R} T_{0}\right)^{0.5}$ and the channel height $H$.

\section{FLOW CONDITIONS}

Computations have been carried out at a fixed pressure ratio $p_{e} / p_{0}=0.9$ and Knudsen number from 0.01 up to 0.1 (slip regime). The dimensionless temperature of the walls is set lower than the inlet temperature $T_{0}$ and equal to $T_{w}=0.9 T_{0}$.

It should be noticed that a fully kinetic solution of the problem would require larger reservoirs [12], while the use of a hybrid solver allows to decrease the reservoirs size down to $L_{x} \times L_{y}=5 \times 3$, significantly decreasing the computational time due to quicker convergence of NS solver in these regions.

In order to accurately model the rough geometry 712 nodes in the streamwise direction (dimensionless minimum grid spacing is 0.004 ) and 40,45 and 50 nodes in the transverse direction for relative roughness height $\varepsilon=5 \%, 2.5 \%$ and $1.25 \%$, respectively, have been used. Minimum transverse grid spacing close to the wall is around 0.003 . Decreasing this grid spacing down to 0.001 introduces maximum differences for the Poiseuille number and the mass flow rate less than $0.8 \%$ and $0.2 \%$, respectively, for both NS and hybrid solvers.

The two-dimensional velocity grid should be selected large enough to capture all of the features of the problem: thus, the velocity space boundary should satisfy the following condition: $v_{\max } \geq \max (|u|,|v|)+4 T_{\max } 0.5$. The number of grid points for each velocity component is 24 and velocity space is bounded by $v_{\max }=5$. The optimal number of grid points in the velocity space was chosen checking that doubling velocity points produces a change in mass flow rate lower than $1-1.5 \%$.

Since the time step is unique for both solvers, it should satisfy the stability (or accuracy) constraint $\Delta t=\min \left(\Delta t_{\mathrm{K}}, \Delta t_{N S}\right)$. The explicit kinetic time step should be limited by the CFL condition with $C F L=0.4$, while $\Delta t_{N S}$ is arbitrary. The solution is considered converged when the criterion $\left\|\mathbf{U}^{n+1}-\mathbf{U}^{n}\right\|_{L 2}<\Delta$ is fulfilled with $L_{2}$ norm and $\Delta=10^{-7}$.

\section{RESULTS AND DISCUSSION}

\section{Heat transfer effect}

Computations at high pressure ratio $p_{e} / p_{0}=0.9$ allows for a nearly incompressible flow condition while varying the rarefaction level and the relative roughness height.

Figure 3 shows the global Nusselt number $N u_{\varepsilon}$ (eq. (12)) as a function of local exit Knudsen number $K n_{e}$ (based on mean free path at the exit of rough section $\lambda_{e}$ and channel height $H$ ) for relative roughness height $\varepsilon$ equal to $5 \%, 2.5 \%, 1.25 \%$ and 0 (smooth), computed using the hybrid solver. It can be seen that the global heat transfer rate, i.e. $N u_{\varepsilon}$, decreases with an increase of rarefaction for both smooth and rough wall channels, as reported in open literature $[7,12,13]$. When the rarefaction increases the local mean free path of gas particles increases resulting in a decrease of interaction between particles; hence, the reduction of global effect of the wall for both rough and smooth channel flows.

Moreover, Figure 3 shows a comparison between the $\mathrm{Nu}$ number computed by the hybrid solver and pure kinetic Smodel results for $\varepsilon=0$ and 5\%: hybrid and kinetic computed 
$N u_{\varepsilon}$ numbers are in a good agreement for both rough $(\varepsilon=5 \%)$ and smooth $(\varepsilon=0)$ channels for the whole range of $K n_{e}$. Thus, the hybrid code can be considered a reliable solver for flow and heat transfer computations over rough fine-textured surfaces.

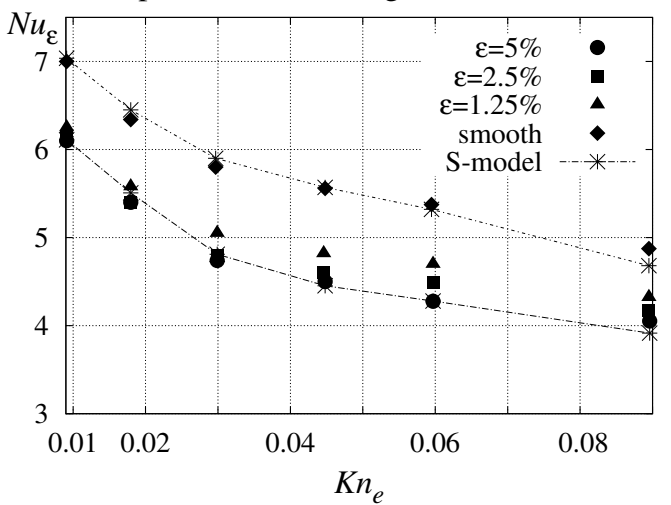

Figure 3 Global Nusselt number $N u_{\varepsilon}$ vs. $K n_{e}$.

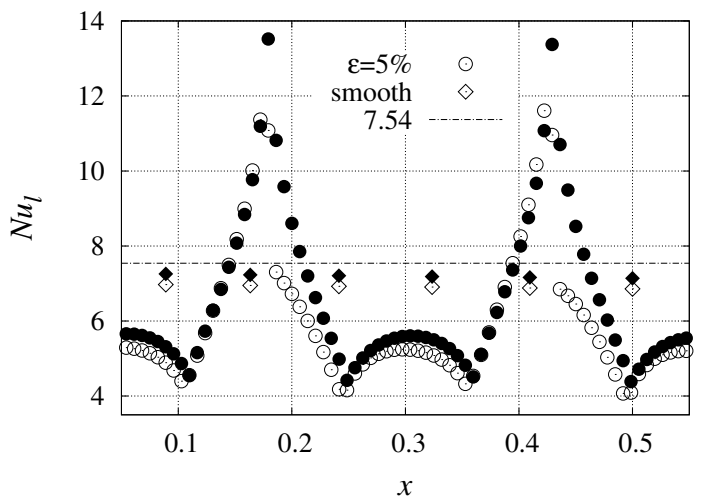

Figure 4 Local Nusselt number $N u_{l}$ along streamwise direction at $K n_{e}=0.009$ : empty symbols, hybrid; solid symbols NS

The wall roughness results in additional decrease of $N u_{\varepsilon}$ in comparison with the smooth channel. For example, at $K n_{e}=0.0091$ this reduction is around $13 \%$. This can be explained if we consider the distribution of local $\mathrm{Nu}$ number along rough and smooth channels (Fig. 4). Apparently, the relatively high value of heat transfer at the top of obstacles cannot compensate the heat reduction within the valley.

Figure 4 shows that within the valleys the discrepancies between NS and hybrid solutions do not exceed 5\%, while around rough peaks there is even a qualitative difference between results. For smooth surface NS and hybrid Nu provide the same results, closer to the analytic value for continuum, incompressible flow of 7.54.

It is interesting to check how the presence of the heat transfer affects other flow parameters, such as global Poiseuille number. For this purpose $P o$ numbers vs. $K n_{e}$ for different relative roughness are shown in Fig. 5: the (cooling) heat transfer decreases $P o$ number in comparison with flow without it: the maximum difference around $5 \%$ is reached for weakly rarefied flow (low Kn number), while for rarefied flow (higher $\mathrm{Kn}$ ) the difference becomes smaller around $2.7 \%$.

Moreover, the increase of the height of wall roughness elements $\varepsilon$ results in an increase of Poiseuille number due to an increase of the wall friction effect. As for the Nusselt number, the increase of rarefaction (Knudsen number) significantly affects the Poiseuille number. Although the Po number for a fixed $K n$ increases as the relative roughness $\varepsilon$ increases, the value of Poiseuille number is larger at lower $K n_{e}$ number and decreases as $K n_{e}$ increases. In particular, for $\varepsilon=5 \%$ the highest $P o_{\varepsilon}=108$ (solid symbols) is for lowest $K n_{e}=0.009$ and the lowest $P o_{\varepsilon}=73$ (solid symbols) for highest $K n_{e}=0.09$.

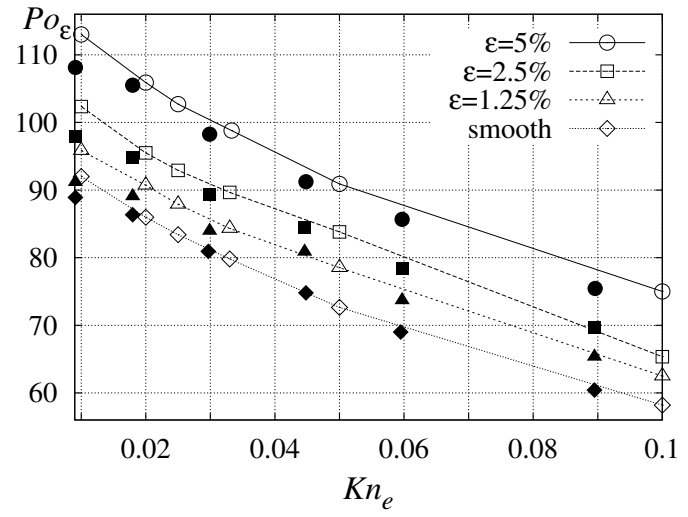

Figure $5 P o_{\varepsilon}$ vs. $K n_{e}$ : empty symbols, without heat transfer; solid symbols, in the presence of heat transfer

As was mentioned before, an increase in rarefaction results in a reduction of friction effect of the wall and hence in a reduction of global Po number for both rough and smooth channel flows. The same decrease of $P o_{\varepsilon}$ with Knudsen number increase has been observed in $[6,8,10]$ as well.

First order boundary conditions accuracy

To quantitatively estimate the applicability of NS solver coupled with the first order slip boundary condition for rough surface modelling (with and without heat transfer) the relative difference between NS and hybrid solutions in terms of global mass flow rate, Poiseuille and Nusselt numbers is computed as:

$$
\Delta \Phi_{h-N S}=1-\frac{\Phi_{N S}}{\Phi_{h}} ; \quad \Phi=W, P o, N u .
$$

The relative differences $\Delta W_{h-N S}, \Delta P o_{h-N S}$ and $\Delta P o_{h-N S}$ in percent are shown in Figs. 6-8 for relative roughness height $\varepsilon$ of zero (smooth channel), $1.25 \%, 2.5 \%$ and $5 \%$.

In the smooth channel NS solver results are in a good agreement with the hybrid ones in the whole range of the Knudsen numbers. With the rough surface we have an appreciable difference between results. Furthermore, the difference in terms of global parameters between hybrid and NS solvers strongly depends on rarefaction level, i.e. $K n_{e}$. The difference in mass flow rate becomes larger than $5 \%$ for $K n_{e}$ around 0.04-0.05 (Fig. 6), in Poiseuille number for $K n_{e}$ around 0.02 . Nusselt number dependence on the wall treatment is even stronger, and the difference $\Delta N u_{h-N S}$ exceeds $5 \%$ already for relatively small $K n$ number, around 0.01 (Fig. 8). The larger difference between solutions in terms of $P O$ number than in mass flow rate is, probably, due to the additional dependence of $P o$ on not only density and velocity, but also viscosity (eq. (9)).

Moreover, from Fig. 6 and 7 the differences continuum and hybrid results, for a given rarefaction level or $K n$ value, are not affected by the presence of heat transfer. The most relevant parameter is therefore the rarefaction level. 


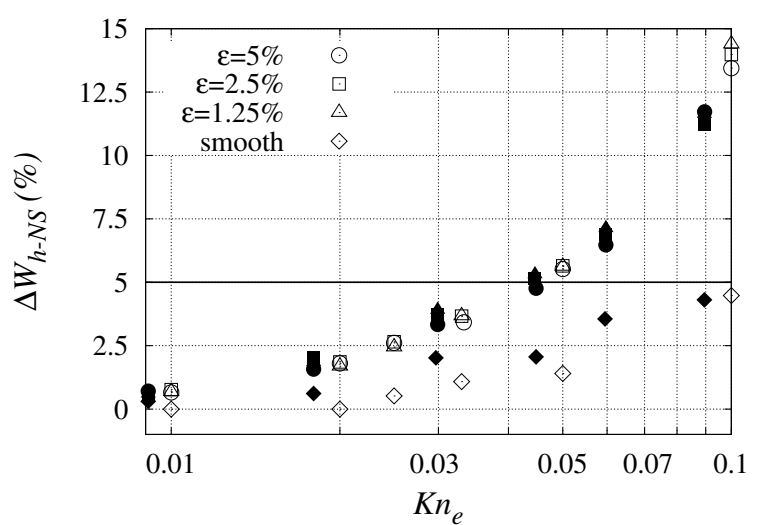

Figure $6 \Delta W_{h-N S}$ vs. $K n_{e}$ : empty symbols, without heat transfer; solid symbols, in the presence of heat transfer.

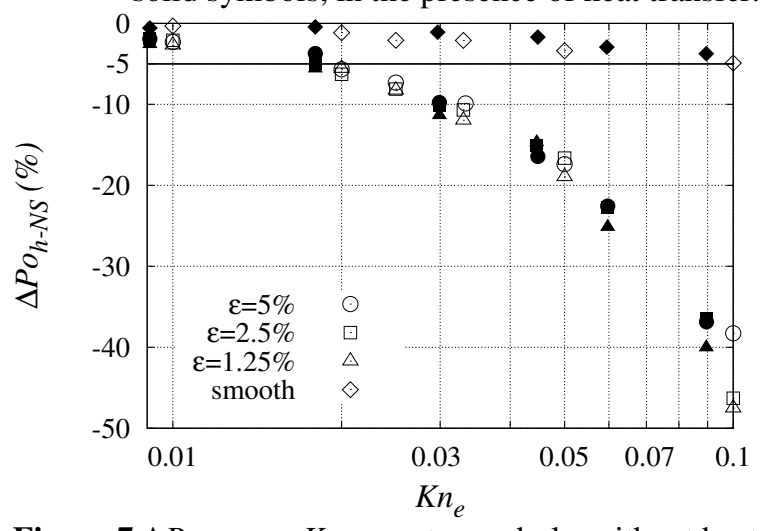

Figure $7 \Delta P o_{h-N S}$ vs. $K n_{e}$ : empty symbols, without heat transfer; solid symbols, in the presence of heat transfer.

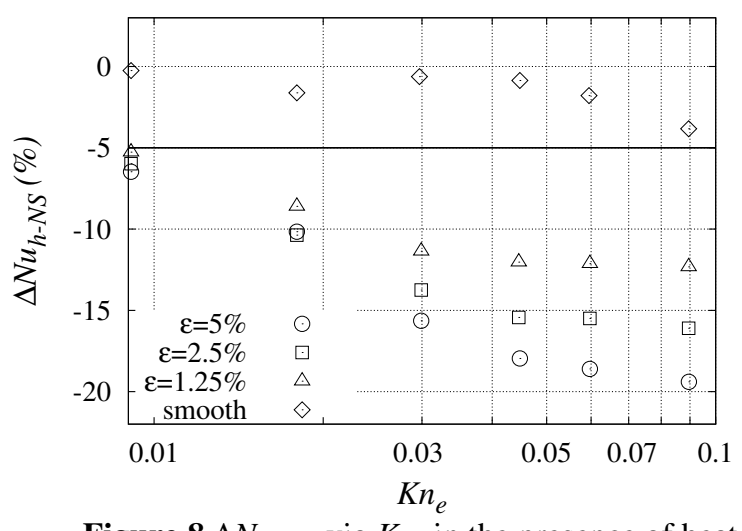

Figure $8 \Delta N u_{h-N S}$ via $K n_{e}$ in the presence of heat transfer.

\section{CONCLUSION}

A hybrid solver, dynamically coupling the direct numerical solution of the S-model kinetic equation with Navier-Stokes equations solution, was applied to investigate the competition between rarefaction, roughness and heat transfer effects in a cooled microchannel. It was found that the roughness has a great effect on the flow characteristic in terms of Poiseuille and Nusselt numbers and mass flow rate. In particularly, the roughness increases $P o$ number, while decreases mass flow rate and $\mathrm{Nu}$ number. On the other hand, an increase in rarefaction results in decrease of $\mathrm{Po}$ and $\mathrm{Nu}$ numbers and mass flow rate for both rough and smooth surfaces due to reduction of wall friction effect. Moreover, if surface roughness is taken into consideration the accuracy of the NS solution provided with first order slip boundary conditions is questionable even for relatively low values of the Knudsen number $(K n=0.01-0.02)$ based on the channel height, probably due to the smaller scale effects related to the roughness peaks, while for a smooth surface the NS solution is reliable for $K n$ up to 0.1 (deviation from kinetic solution does not exceed 5\%).

\section{REFERENCES}

[1] Degond P., Dimarco G., and Mieussens L., A moving interface method for dynamic kinetic-fluid coupling, Journal of Computational Physics, Vol. 227, 2007, pp. 1176-1208

[2] Rovenskaya O., Croce G., Coupling kinetic and continuum equations for micro scale flow computations, Heat Transfer Engineering, Vol 34, No. 2-3, 2013, pp. 192-203

[3] Darbandi M., and Roohi E., A hybrid DSMC/Navier-Stokes frame to solve mixed rarefied/nonrarefied hypersonic flows over nanoplate and micro-cylinder, Int. J. for Numerical Methods in Fluids, Vol. 72, 2013, pp. 937-966

[4] Rovenskaya O., and Croce G., Application a hybrid solver to gas flow through a slit at arbitrary pressure ratio, Vacuum, Vol. 109, 2014, pp. 266-274

[5] Rovenskaya O., and Croce G., Application of the hybrid kineticcontinuum solver to the near wall modelling Journal of Physics: Conference Series, Vol.547, 2014, pp. 012020

[6] Rovenskaya O., and Croce G., Numerical investigation of microflow over rough surfaces: coupling approach Journal of Heat Transfer, Vol. 135, 10, 2013, pp. 101005

[7] Croce G., and D'Agaro P., Compressibility and rarefaction effect on heat transfer in rough microchannels, Int. J. of Thermal Sciences, Vol. 48, 2009, pp. 252-260

[8] Zhang C., Chen Y., and Shi M., Effects of roughness elements on laminar flow and heat transfer in microchannels, Chemical Engineering and Processing: Process Intensification, Vol. 49, No 11, 2010, pp. 1188-1192

[9] Sun H., and Faghri M., Effect of surface roughness on nitrogen flow in a microchannel using the direct simulation Monte Carlo method, Numerical Heat Transfer Part A, Vol. 43, 2003, pp. 1-8.

[10] Rovenskaya O.I., Kinetic analysis of surface roughness in a microchannel, Computers \& Fluids, Vol. 77, 2013, pp. 159-165

[11] Ji Y., Yuan K., and Chung J., Numerical simulation of wall roughness on gaseous flow and heat transfer in a microchannel, Int. J. of Heat and Mass Transfer, Vol. 49, 2006, pp. 1329-1339.

[12]Zhang, WM, Meng, G, Wei XY, Peng, ZK, Slip flow and heat transfer in microbearings with fractal surface topographies, Int. J. of Heat and Mass Transfer, Vol.55, 2012, 7223-7233

[13] Khadem MH, Shams M, Hossainpour S, Numerical simulation of roughness effects on flow and heat transfer in microchannels at slip flow regime, Int. Comm. Heat \& Mass Transfer, Vol.36, 2009, 69-77

[14] Rovenskaya O., Comparative analysis of the numerical solution of full Boltzmann and BGK model equations for the Poiseuille flow in a planar microchannel, Comp. \& Fluids, Vol. 81, 2013, pp. 45-56.

[15] Shakhov E.M., A method for calculating rarefied gas flows. Moscow, Nauka, 1974.

[16] Kolgan V.P., Application of the principle of minimizing the derivative to the construction of finite-difference schemes for computing discontinuous solutions of gas dynamics, Journal of Computational Physics, Vol. 230, No. 7, 2011, pp. 2384-2390.

[17] Pulliam T.H., Artificial dissipation models for the Euler equations, AIAA Journal, Vol. 24, 1986, pp. 1931-1940.

[18] Chapman S., and Cowling T.G., The mathematical theory of nonuniform gases, 3rd edition, Cambridge University Press, 1990. 